\title{
Optical Image Quality Improvement Based on Time-resolved Stokes Vectors in Filamentous Tissues
}

\author{
Chia-Wei Sun, C. C. Yang, and Yean-Woei Kiang \\ Graduate Institute of Electro-Optical Engineering, Graduate Institute of Communication Engineering \\ and Department of Electrical Engineering, National Taiwan University, \\ 1, Roosevelt Road, Section 4, Taipei, Taiwan \\ (phone) 886-2-23657624 (fax) 886-2-23652637 (E-mail) ccy@cc.ee.ntu.edu.tw
}

\begin{abstract}
We first demonstrated the effectiveness of imaging in a tissue phantom with isotropic scattering by using polarization discrimination combined with the time gating method. In this situation with lean pork as targets and diluted milk as tissue phantom, the reduced scattering coefficient mapping manifests clear images. However, such an imaging technique became less effective in filamentous tissues, such as chicken breast tissues, because filamentous tissue had a deterministically anisotropic property. It led to coherent coupling between the two linear polarization components. In this situation, we employed the time-gated degree of polarization (DOP) imaging technique that based on the Stokes formalism. The results showed that the DOP measurement was quite effective in high-quality imaging of objects in filamentous tissues. The improvement of this method was attributed to the unchanged polarization part under the coupling processes of various polarization components.
\end{abstract}

Keywords: time gating, polarization discrimination, optical imaging, filamentous tissue, degree of polarization

\section{INTRODUCTION}

Recently, biomedical imaging based on optical techniques has become an active area of research for developing medical diagnosis techniques. For thick biological tissues of several $\mathrm{cm}$, ultrafast-optics techniques have been considered for optical imaging based on time-gated transmitted signals. In such a technique, psec or fsec pulses are applied to tissues and the transmitted quasi-coherent photons (snake photons) are extracted. While time-gating methods have been proved useful for biomedical imaging, the polarization discrimination method is another important one [1-5]. This method is based on the depolarization effect in random scattering of tissues. In this effect, the input polarization state is partially preserved in weakly scattered photons. Thus, the quasi-coherent part can be extracted by transmitted co-polarized photons. However, the measurement of the depolarization effect by discriminating coherent from incoherent photons becomes less effective when there exists coherent coupling between two perpendicular polarization components. Such coherent coupling phenomena can particularly occur in filamentous tissues with anisotropic structures. In this situation, the conventional polarization gating method becomes ineffective in optical imaging. However, we have found that by using the Mueller-Stokes formalism, images of higher contrast and resolution in filamentous tissues could be achieved. In other words, images of the degree of polarization (DOP), based on the measurement of the Stokes vectors, manifest quite high quality in filamentous tissues. In this paper, we report the developments of the aforementioned imaging techniques based on polarization manipulations of the transmitted signal through tissue samples in our laboratory. We will first show the advantages of time-gating and polarization discrimination in imaging objects in an isotropic turbid medium. Diluted milk was used as the medium for isotropic scattering. Then, we used chicken breast tissues, consisting of filaments with optically isotropic I bands and anisotropic A bands, as samples for demonstrating the failure of imaging in filamentous tissues when the aforementioned technique of combining time gating and polarization discrimination is used. Finally, the DOP was calibrated based on the measurements of the Stokes vectors for effective imaging in a filamentous tissue structure.

\section{POLARIZATION-DEPENDENT CHARACTERISTICS IN FILAMENTOUS TISSUES}

The image results have indicated that the combination of polarization-discrimination with the time-gating method improves image contrast in an isotropic tissue phantom. For the study in filamentous tissues, we used chicken breast tissues as samples in our experiments. The chicken breast tissue sample has the thickness varying from 1.3 to $1.5 \mathrm{~cm}$ within the transverse scanning area. The imaging results with a thin chicken bone of diameter 1-2 $\mathrm{mm}$ (as the object) sticking into the breast tissue sample with the input linear polarization along the tissue filament direction are shown in Fig. 1(a) and (b). Parts (a) and (b) 
show the images of $I_{p}$ and $I_{p}-I_{c}$, respectively. The result of $I_{p}$ provides a rough picture of the chicken bone; however, $I_{p}-I_{c}$ leads to no feature at all. The imaging results confirm the coherent polarization evolution besides random scattering in skeletal muscle tissues. This indicates that besides possible random scattering in such a medium, coherent coupling between the two polarization components occurs due to the organized structure of the filamentous tissue. Due to certain coherent cross-polarized interactions, signal polarization seemed to rotate such that $I_{c}$ became higher than $I_{p}$ at the output. In this situation, both $I_{p}$ and $I_{c}$ were quite coherent, particularly before and near their peaks. Hence, the conventional polarization gating technique, i.e., imaging with $I_{p}-I_{c}$, became difficult.

\section{TIME-RESOLVED STOKES VECTOR AND DOP IMAGING IN FILAMENTOUS TISSUES}

As discussed above, deterministic optical birefringence results in coherent polarization evolution, leading to coherent polarization coupling and hence invalid coherent photon extraction through polarization discrimination. In this situation, all the elements of polarization state must be considered for understanding the polarization evolution and hence improving image quality in such a medium. In this section, we intent to apply the Stokes formalism for improving image contrast in the filamentous tissue. According to the Stokes formalism, we need to measure four different polarization states of transmitted photons, including horizontal linear polarization component $H$, vertical linear polarization component $V, 45^{\circ}$ linear polarization component $P$, and right-hand circular polarization component $R$. In the experiments, such polarization control was accomplished through adjusting a polarizer and a quarter-wave plate. Here, the horizontal and vertical polarization directions are referred to the coordinate of the laboratory. The quasi-coherent photon data were obtained by gating the time-resolved intensity profiles with duration of $50 \mathrm{psec}$ from the leading edges of the temporal curves.

The Stokes components, generally denoted by $S_{0}, S_{1}, S_{2}$ and $S_{3}$, form a sufficient set for describing the amplitude, phase and polarization of a light wave. They are proportional to the total intensity, the difference between the horizontal and vertical polarization components, the difference between the linearly polarized components oriented at $45^{\circ}$ and $-45^{\circ}$, and the subtraction of the left-handed from the right-handed circularly polarized components, respectively. Subsequently, we can calculate the DOP as follow:

$$
D O P=\frac{\sqrt{S_{1}^{2}+S_{2}^{2}+S_{3}^{2}}}{S_{0}} .
$$

Again, we used chicken breast tissues of $1.5 \mathrm{~cm}$ in thickness as filamentous tissue samples in the experiments.

Figure 2 shows the time-resolved Stokes parameters of chicken breast tissue with the filaments along the horizontal polarization direction. The results were obtained with horizontally polarized incident pulses. The $S_{0}$ profile represents the total intensity of transmitted signal that contains quasi-coherent and diffuse photons. The $S_{l}$ profile is featureless because the mixed effects of coherent polarization coupling and random de-polarization process just result in almost cancellation between the $H$ and $V$ components in this case. Such featureless $S_{l}$ was the major reason for ineffective imaging operation based on polarization discrimination. On the contrary, the $S_{2}$ and $S_{3}$ profiles furnish the information of coupled polarization components of quasi-coherent photons. The depression features of $S_{2}$ and $S_{3}$ near the leading edges of the time-resolved profiles should result in more image information if the time-gated Stokes vector is calibrated.

To demonstrate the improvement of image contrast based on the time-gated Stokes vector, we stuck a thin chicken bone (its shape and size are shown in Fig. 3) into the chicken breast tissue, with bone elongation in the vertical polarization direction for optical imaging. Line scan images were obtained by linear scanning in the direction perpendicular to the bone elongation, i.e., scanning across the bone. The spatial range in the abscissa is $1.1 \mathrm{~cm}$ with the step size of $1 \mathrm{~mm}$. The gray level represents the relative intensity distribution. In Fig. 3, we compare various gating schemes, including (a) time gated integrated intensity $\left(S_{0}\right)$, (b) time-gated $S_{l}$ component, i.e., the conventional approach of combining polarization discrimination with time gating, (c) DOP distribution with time gating, and (d) DOP distribution without time gating. As shown in part (d), even a rough image cannot be obtained without time gating. The comparison between parts (a)-(c) shows that although images of the chicken bone were obtained with time gating and/or polarization discrimination, an image of higher resolution and contrast was achieved with DOP evaluation. This result implies that the time-gated DOP measurement can substitute the conventional polarization-discrimination method in filamentous tissues for optical imaging.

\section{CONCLUSIONS}

In this paper, we first discussed the effectiveness of imaging in a tissue phantom with isotropic scattering by using polarization discrimination combined with time gating. In this situation with pork as target and diluted milk as tissue phantom, the reduced scattering coefficient mapping manifests clear images. However, such an imaging method became less effective in filamentous tissues, such as chicken breast tissues because filamentous tissue had a deterministically anisotropic property. It led to coherent coupling between the two linear polarization components. The coherent coupling resulted in difficulty of using the conventional polarization gating method for optical imaging of filamentous tissues. In this situation, we employed the time-gated DOP imaging technique that based on the Stokes formalism. The results showed that the DOP measurement 
was quite effective in high-quality imaging of objects in filamentous tissues. The improvement of this method was attributed to the unchanged polarization part under the coupling processes of various polarization components. Because the Stokes vector provides complete polarization information of transmitted light, this technique can be applied to imaging and characterization of other kinds of filamentous tissues.

\section{ACKNOWLEDGEMENTS}

This research was supported by National Health Research Institute, The Republic of China, under the grant of NHRI-GT-EX89, and by National Science Council, The Republic of China, under grants of NSC 89-2218-E-002-094 and NSC 89-2218-E-002-095.

\section{REFERENCES}

[1] Schmitt J. M., Gandjbakhche A. H., and Bonner R. F.: Use of polarized light to discriminate short-path photons in a multiply scattering medium. Appl. Opt. 1992; 31, 6535-6546.

[2] Horinaka H., Hashimoto K., Wada K., Cho Y., and Osawa M.: Extraction of qusai-straightforward-propagating photons from diffused light transmitting through a scattering medium by polarization modulation. Opt. Lett. 1995; 20, 1501-1503.

[3] Demos S. G. and Alfano R. R.: Temporal gating in highly scattering media by the degree of optical polarization. Opt. Lett. 1996; 21, 161-163.

[4] Morgan S. P., Khong M. P., and Somekh M. G.: Effects of polarization state and scatterer concentration on optical imaging through scattering media. Appl. Opt. 1997; 36, 1560-1565.

[5] Sankaran V., Walsh J. T., and Maitland D. J.: Polarized light propagation through tissue phantoms containing densely packed scatterers. Opt. Lett. 2000; 25, 239-241.

(a)

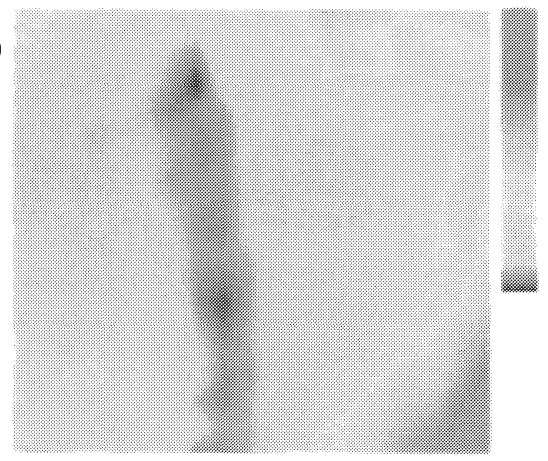

(b)

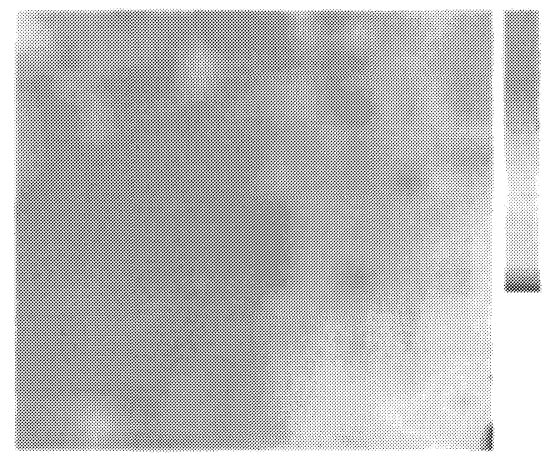

Fig. 1 Time-gated images of a chicken bone in chicken breast tissue with (a) $I_{p}$ and (b) $I_{p}-I_{c}$.

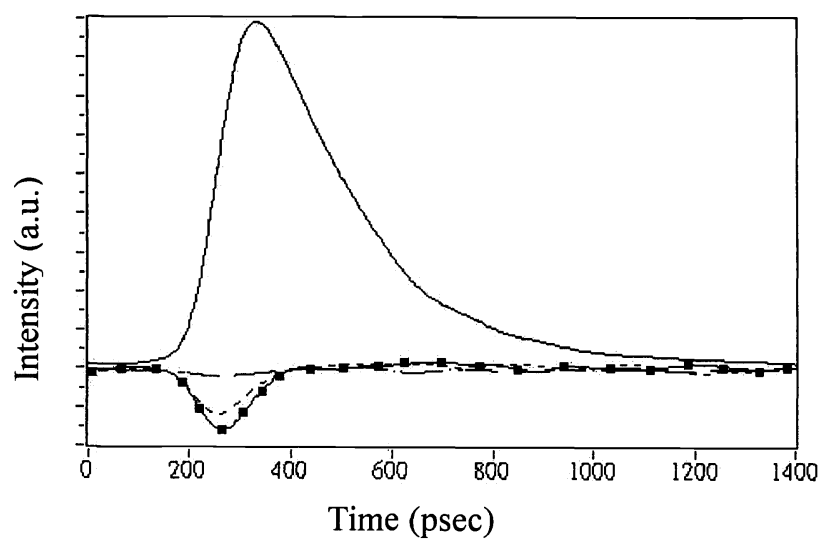

Fig. 2 Time-resolved Stokes element profiles of chicken breast tissue. 


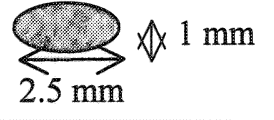

(a)

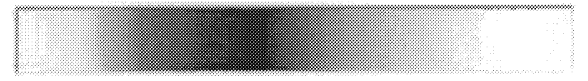

(b)

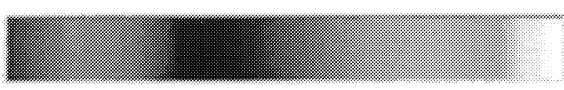

(c)

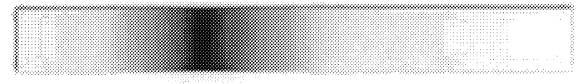

(d)

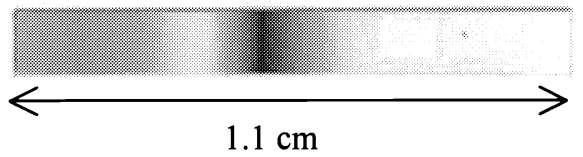

Fig. 4 1-D images of the chicken bone in chicken breast tissue with filament orientation in the horizontal direction: (a) time-gated integrated intensity $\left(S_{0}\right)$, (b) time-gated $S_{l}$ component, (c) DOP with time gating, and (d) DOP without time gating. 